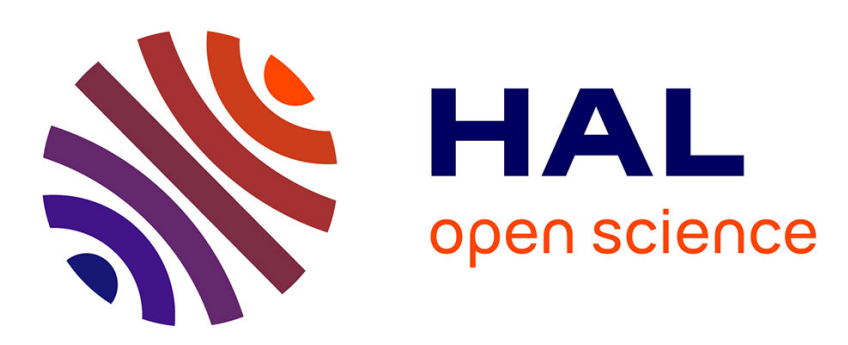

\title{
Bayesian model identification of higher-order frequency response functions for structures assembled by bolted joints
}

\author{
Rafael Teloli, Samuel da Silva, Thiago G Ritto, Gael Chevallier
}

\section{To cite this version:}

Rafael Teloli, Samuel da Silva, Thiago G Ritto, Gael Chevallier. Bayesian model identification of higher-order frequency response functions for structures assembled by bolted joints. Mechanical Systems and Signal Processing, 2021, 151, pp.107333 (17). hal-03359913

\section{HAL Id: hal-03359913 https://hal.science/hal-03359913}

Submitted on 30 Sep 2021

HAL is a multi-disciplinary open access archive for the deposit and dissemination of scientific research documents, whether they are published or not. The documents may come from teaching and research institutions in France or abroad, or from public or private research centers.
L'archive ouverte pluridisciplinaire HAL, est destinée au dépôt et à la diffusion de documents scientifiques de niveau recherche, publiés ou non, émanant des établissements d'enseignement et de recherche français ou étrangers, des laboratoires publics ou privés. 


\title{
Bayesian Model Identification of Higher-Order Frequency Response Functions for Structures Assembled by Bolted Joints
}

\author{
Rafael de O. Teloli, Samuel da Silva \\ ${ }^{1}$ UNESP - Universidade Estadual Paulista, Faculdade de Engenharia de Ilha Solteira, \\ Departamento de Engenharia Mecânica, Av. Brasil, 56, Ilha Solteira, 15385-000, SP, Brasil \\ Thiago G. Ritto \\ 2 UFRJ - Universidade Federal do Rio de Janeiro, Departamento de Engenharia Mecânica, \\ Ilha do Fundão, Rio de Janeiro, 21945-970, RJ, Brasil \\ Gaël Chevallier \\ 3 Univ. Bourgogne Franche-Comté, FEMTO-ST Institute, CNRS/UFC/ENSMM/UTBM, \\ Department of Applied Mechanics, 24 chemin de l'Epitaphe, 25000 Besancon, France.
}

\begin{abstract}
This paper proposes a procedure to identify a stochastic Bouc-Wen model for describing the dynamics of a structure assembled by bolted joints considering vibration data. The proposed identification approach is expressed into a Bayesian framework to take into account the data fluctuations related to uncertainties in the measurement process. The calibration of the model parameters uses the analytical expressions of the higher-order frequency response functions (FRFs) for approximating experimental measurements. The Metropolis-Hastings algorithm is employed for approximating posterior distributions. Once calibrated, the applicability of the probabilistic Bouc-Wen model is evaluated, and its dynamical behavior is compared with experimental measurements from the bolted structure. The results show that the stochastic version of the Bouc-Wen model can predict with adequate agreement, including hysteretic effects, the output of the jointed structure considering several excitation amplitudes.
\end{abstract}

Keywords: hysteretic systems, higher-order frequency response function, Volterra series, jointed structures, Bayesian identification

\footnotetext{
*Rafael de Oliveira Teloli

Email addresses: rafael.teloli@unesp.br (Rafael de O. Teloli), samuel.silva13@unesp.br (Samuel da Silva), tritto@mecanica.coppe.ufrj.br (Thiago G. Ritto), gael.chevallier@femto-st.fr (Gaël Chevallier)
} 


\section{Introduction}

Several engineering systems are built with components assembled through different types of joints or mechanical connections, which may affect the dynamic performance of such structures. Concerning systems assembled by bolted joints,

5 the frictional contact between different structural components under vibrational loading cycles leads to the appearance of the hysteresis effect due to the stickslip motion occurring over the connected area [1]. In this context, developing predictive numerical models of jointed structures is not an easy task, quite the opposite. These systems may undergo challenging nonlinear behavior over their operating range, including softening stiffness mechanism which decreases the resonant frequency at high excitation amplitudes, multi-valued hysteretic restoring forces, or even memory dependency on the input, output and an evolutionary variable that induces a delay among them [2, 3, 4. Several hysteretic models carry the potential to characterize such nonlinearities, including the

15 Iwan, Bouc-Wen, LuGre, Valanis, Coulomb, Masing, Jenkins models, etc. [5]. The scientific community that investigates the dynamics of jointed structures is very active at the moment, and still searching for scientific contributions toward modeling and identification fields.

Note that frequency domain formulations are advantageous in dealing with assembled structures. One topic of interest involves the identification of the evolution of resonance frequencies and damping ratios, according to excitation amplitudes, the so-called "modal backbone" curves. Peyret et al. [6] developed a framework for tracking of experimental backbones involving a clamped-clamped beam with transverses interfaces subject to friction and partial slip. The au-

25 thors post-processed the modal damping and resonance frequencies related to the first bending mode of a by using the extended Kalman filter. Based on the concept of nonlinear modes, Scheel et al. 7] developed a force appropriation technique, which considers steady-state response periods for extracting resonance frequencies and damping ratios considering isolated modes. Then, these so modal parameters are used to construct the modal backbone curve. Alternatively to these curves, frequency-domain methods are often used for predicting the nonlinear behavior of assembled structures. Jaumouillé et al. 8] proposed an adaptive harmonic balance method to compute higher-order harmonic amplitudes of a bolted joint structure. Claeys et al. [9] implemented the harmonic 35 balance method with continuation and condensation algorithms to predict the nonlinear vibrational response of an experimental setup, which includes frictional elements. Lacayo et. al [10] put forward a frequency-domain solver, which is based on a multi-harmonic balance approach, for capturing the slight amplitude-dependency behavior of a bolted lap-joint structure.

40 The frequency-domain techniques are also useful in parameter estimation procedures. In this context, the multidimensional Fourier transform of the Volterra Kernels is a possible approach to apply. The so-called higher-order frequency response functions (FRFs) generalize the concept of the FRFs for nonlinear systems exploiting the convolution structure on these kernels [11, 12. 45 The computation of the higher-order FRFs is performed having the mathemat- 
ical model of the system or structure in interest by using the harmonic probing method, which consists of exciting the nonlinear system with a proper combination of harmonic inputs [13]. On the one hand, the framework of the higher-order FRFs is suitable for applications involving parameter estimation on polynomial 50 nonlinearities [14, 15, 16. However, there is a lack of contributions related to parameter estimation of hysteretic systems. Recently, Teloli and da Silva [17. introduced a new approach for harmonic probing of hysteretic systems through a nonlinear smooth operator. The authors predicted in a pioneering way, using closed-form solutions, the output of a Bouc-Wen oscillator after rewriting the 55 loading and unloading regimes of the hysteresis loop through smooth operators, which are based on the Taylor series procedure.

In the present paper, we use a stochastic version of this Bouc-Wen model to reconstruct a probabilistic hysteresis loop of the first vibrating mode of an experimental cantilever beam formed of two aluminum beams assembled by a bolt connection considering data variation related to the presence of uncertainties in the measurement process. It is essential to point out that the hysteretic model is not used to describe directly the frictional forces in contact interfaces, but rather the whole restoring forces related to the mode of interest. The BoucWen model stands out for accomodating a wide range of hysteresis loops in 65 the restoring force versus displacement plane [18. Additionally, it is argued that this model can represent any Iwan's model, which is the most widely used model for modeling jointed structures, just by selecting its parameters [19]. It is worth mentioning the work of Oldfield et al. [20, which brings forward a Bouc-Wen model for producing the hysteresis loop generated by synthetic data 70 of a finite element model involving three components in the assembled configuration. However, the fact that the Bouc-Wen parameters are non-physical turns the parameter identification process into a challenging task if not properly conducted, and justifies why their use for capturing the dynamic behavior in jointed structures is still modest [21].

75 Thus, with regard to the parameter identification scheme to compute the Bouc-Wen parameters, this work proposes a procedure formulated into a whitebox Bayesian modeling framework and considers, as an essential step in the complete identification process, the use of higher-order FRFs derived by Teloli and da Silva [17. The advantage of using the Volterra kernels at this idenso tification scheme lies in the possibility of visualizing, with physical meaning, the influence of the nonlinear parameters on the system output through the Volterra contributions. The main contribution of this work lies in proposing a new parameter estimation procedure in reduced numerical probabilistic models for representing the dynamics of bolted joint structures by propagating uncer${ }_{85}$ tainties in the coefficients that control the hysteresis loop. Moreover, although this paper investigates the first vibrating mode of a structure which carries complex nonlinear effects, the identification strategy here proposed may be suitable for dealing with hysteretic systems that potentially allow the use of smoothing procedures, such as the bit-rock interaction of a drill string 22 or even

90 as a formulation in the parametric reduced-order models of jointed structures [23, 24]. 
Toward this background, the parametric identification strategy here proposed includes, firstly, the identification of the underlying linear model using conventional modal techniques. Then, the higher-order FRFs are used to calibrate the Bouc-Wen parameters with independent experimental realizations with probing tests. After, through the Markov Chain Monte Carlo (MCMC) Metropolis-Hastings algorithm, the probability density function (PDF) of each random variable is updated. Once calibrated, the computational model is evaluated, and its dynamical behavior is compared with experimental measurements

100 from the bolted structure. For covering all these steps, the paper's outline is section 2 presents an overview of the smoothing procedure carried on the Bouc-Wen oscillator and also the theoretical background for deriving the higherorder FRFs through the harmonic probing method. Section 3, in turn, starts presenting a complete description of the parameter estimation procedure proposed. Then the section goes on the Bayesian inference strategy, describing the MCMC/Metropolis-Hastings algorithm to update the random variables. Next, section 4 presents an experimental application of the identification procedure for identifying an assembled structure by bolted joints, exemplifying the applicability of the stochastic Bouc-Wen oscillator in representing the linear and 110 nonlinear behavior of the experimental test-bench structure. Since this model is not commonly used to represent bolted joints, comparing with the Iwan's model, this paper shows that the Bouc-Wen model operates well, assuming the available experimental data. Finally, section 5 reports the final remarks and the next steps for future work.

\section{Overview of the Higher-Order FRFs for Hysteretic Systems}

\subsection{Smoothing of the deterministic Bouc-Wen model}

The single-degree-of-freedom Bouc-Wen model is given by:

$$
\begin{array}{r}
\ddot{y}(t)+2 \zeta \omega_{n} \dot{y}(t)+k y(t)+\mathcal{Z}(y, \dot{y})=\tilde{u}(t) \\
\dot{\mathcal{Z}}(y, \dot{y})=\alpha \dot{y}(t)-\gamma|\dot{y}(t)||\mathcal{Z}(y, \dot{y})|^{\nu-1} \mathcal{Z}(y, \dot{y})-\delta \dot{y}(t)|\mathcal{Z}(y, \dot{y})|^{\nu}
\end{array}
$$

where $\omega_{n}$ is the resonance frequency, $\zeta$ is the damping ratio, $k$ is a normalized stiffness coefficient and $\ddot{y}(t), \dot{y}(t)$ and $y(t)$ are the acceleration, velocity and displacement, respectively, of the hysteretic oscillator when subjected to an input $\tilde{u}(t)$, whereas $\mathcal{Z}(y, \dot{y})$ is the hysteretic restoring force that obeys the differential term $\dot{\mathcal{Z}}(y, \dot{y}) ; \alpha, \gamma, \delta$ and $\nu$ are the Bouc-Wen parameters. For this paper, $\nu=1$.

Figure 1(a) exemplifies, in the restoring force $\times$ displacement plane, the hysteresis loop produced by a Bouc-Wen model when subjected to a bounded ${ }_{125}$ harmonic input $\tilde{u}(t)=A \cos (\omega t)$, defined in a period $T \in\left[\begin{array}{ll}0 & \frac{2 \pi}{\omega}\end{array}\right]$, whereas Fig. 1 (b) depicts an exemplifying output. The hysteretic restoring force $\mathcal{Z}(y, \dot{y})$ is a multi-valued function according to the excursion interval of the pair $(\mathcal{Z}, y)$, which can move along the path $A C D$ when $\dot{\tilde{u}}<0$, otherwise it moves on the path $D B A$ for $\dot{\tilde{u}}>0$; these excursion intervals define the loading and unloading 


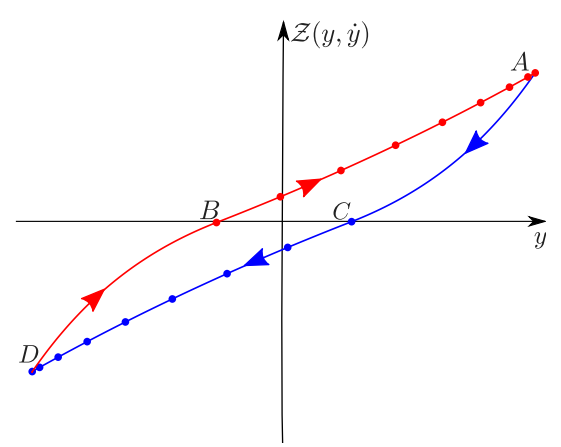

(a) Hysteresis loop of a hysteretic model

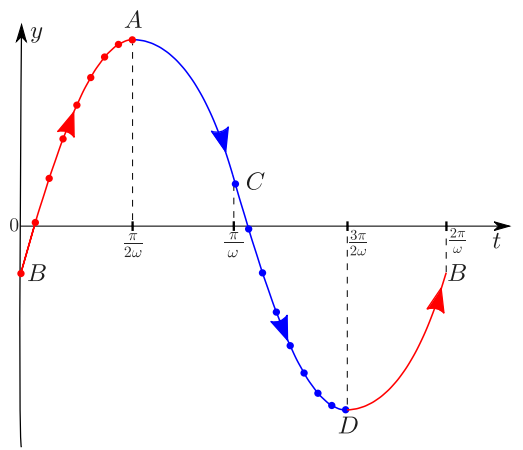

(b) Exemplifying hysteretic output.

Figure 1: Illustrative example of the hysteresis loop. — represents unloading regime of motion whereas _ represents the loading one.

Additionally, the hysteretic restoring force of the Bouc-Wen model allows its division into four different paths. Teloli and da Silva [17 have shown that for conditions where the hysteresis force is weak, each of these paths can be smoothed by a functional expansion through the Taylor series approach:

- path AC: $\dot{y} \leqslant 0, \mathcal{Z} \geqslant 0$

$$
\mathcal{Z}_{1} \approx \frac{\alpha}{(\delta-\gamma)}\left(1-\left[\sum_{n=0}^{\infty} \frac{[-(\delta-\gamma)]^{n}\left(y-y_{0}\right)^{n}}{n !}\right]\right)
$$

- path CD: $\dot{y} \leqslant 0, \mathcal{Z} \leqslant 0$

$$
\mathcal{Z}_{2} \approx-\frac{\alpha}{(\delta+\gamma)}\left(1-\left[\sum_{n=0}^{\infty} \frac{[(\delta+\gamma)]^{n}\left(y-y_{0}\right)^{n}}{n !}\right]\right)
$$

- path DB: $\dot{y} \geqslant 0, \mathcal{Z} \leqslant 0$

$$
\mathcal{Z}_{3} \approx-\frac{\alpha}{(\delta-\gamma)}\left(1-\left[\sum_{n=0}^{\infty} \frac{[(\delta-\gamma)]^{n}\left(y+y_{0}\right)^{n}}{n !}\right]\right)
$$

- path BA: $\dot{y} \geqslant 0, \mathcal{Z} \geqslant 0$

$$
\mathcal{Z}_{4} \approx \frac{\alpha}{(\delta+\gamma)}\left(1-\left[\sum_{n=0}^{\infty} \frac{[-(\delta+\gamma)]^{n}\left(y+y_{0}\right)^{n}}{n !}\right]\right)
$$

where the pair $\left(\mathcal{Z}_{1}, \mathcal{Z}_{2}\right)$ corresponds to the unloading cycle of the hysteresis loop, whereas the pair $\left(\mathcal{Z}_{3}, \mathcal{Z}_{4}\right)$ is for the loading one. Although the more terms included in the Taylor series approach, the more significant the precision of the polynomial form for the hysteresis loop will be; the conditions addressed in this work deliver perfectly acceptable results assuming the first three orders $n=3$ 
for each path [17. A similar procedure for smoothing nonlinear systems was performed by Miguel et. al 25.

Based on the Weierstrass approximation theorem [26], it is suggested the following bounding functions $\mathscr{F}^{\uparrow}[y(t)]$ and $\mathscr{F} \downarrow[y(t)]$, both limited along the interval $y_{\min } \leq y(t) \leq y_{\max }$, to describe properly the load and unload regime:

$$
\begin{array}{cccc}
\mathscr{F}^{\uparrow}[y(t)]=\lambda_{0}+\lambda_{1} y(t)-\lambda_{2} y^{2}(t)+\lambda_{3} y^{3}(t) & \text { for } & \operatorname{sign}[\dot{\tilde{u}}(t)] \geq 0 \\
\mathscr{F}^{\downarrow}[y(t)]=-\lambda_{0}+\lambda_{1} y(t)+\lambda_{2} y^{2}(t)+\lambda_{3} y^{3}(t) & \text { for } & \operatorname{sign}[\dot{\tilde{u}}(t)] \leq 0
\end{array}
$$

where $\lambda_{0}\left[\mathrm{Nkg}^{-1}\right], \lambda_{1}\left[\mathrm{Nkg}^{-1} / \mathrm{m}\right], \lambda_{2}\left[\mathrm{Nkg}^{-1} / \mathrm{m}^{2}\right]$ and $\lambda_{3}\left[\mathrm{Nkg}^{-1} / \mathrm{m}^{3}\right]$ are the 150 bounding function coefficients. Due to the symmetry of the hysteresis loop, the bounding functions present an equivalent structure, which means that the coefficients with the same subscript have equal absolute values. Nevertheless, the function $\mathscr{F} \uparrow[y(t)]$ is responsible for encoding the loading paths $\left(\mathcal{Z}_{3}, \mathcal{Z}_{4}\right)$, whereas $\mathscr{F} \downarrow[y(t)]$ encodes $\left(\mathcal{Z}_{1}, \mathcal{Z}_{2}\right)$. The polynomial order is selected according to the order used in the Taylor series approach [17.

Thus, the coefficients of the polynomial form are expressed as a function of the Bouc-Wen parameters by minimizing an error function described by:

$$
E\left(\lambda_{0}, \lambda_{1}, \lambda_{2}, \lambda_{3}\right)=\int_{-Y}^{y_{0}}\left\{\mathcal{Z}_{2}-\mathscr{F}^{\downarrow}[y(t)]\right\}^{2} d y+\int_{y_{0}}^{Y}\left\{\mathcal{Z}_{1}-\mathscr{F} \downarrow[y(t)]\right\}^{2} d y
$$

subjected to $\frac{\partial E}{\partial \lambda_{i}}=0$, for $i=0,1,2,3$. Minimization procedure results in the following expressions:

$$
\begin{array}{r}
\lambda_{0}=\frac{\alpha y_{0}}{16}\left(3 \delta Y+8 \gamma y_{0}-16\right) \\
\lambda_{1}=\alpha \\
\lambda_{2}=\frac{\alpha}{16 Y}\left(8 \delta^{2} Y y_{0}+8 \gamma^{2} Y y_{0}+15 \delta \gamma y_{0}^{2}-8 \gamma Y-15 \delta y_{0}\right) \\
\lambda_{3}=\frac{\alpha}{96 Y^{5}}\left(\begin{array}{c}
16 \delta^{2} Y^{5}+70 \delta \gamma Y^{4} y_{0}-70 \delta \gamma Y^{2} y_{0}^{3}-35 \delta Y^{4}+ \\
105 \delta Y^{2} y_{0}^{2}-105 \delta y_{0}^{4}
\end{array}\right)
\end{array}
$$

where $Y=\left|y_{\min }\right|=\left|y_{\max }\right|$ and $y_{0}$ is a threshold displacement. These equations are valid only for harmonic excitations that ensure a weak hysteretic force and when the force $\times$ displacement plane draws a single loop.

The switch between bounding functions occurs according to $\operatorname{sign}[\dot{\tilde{u}}(t)]$ and thus, an equivalent system with hysteresis is proposed:

$$
\ddot{y}(t)+2 \zeta \omega_{n} \dot{y}(t)+k y(t)+\underbrace{u_{0}(t)+\lambda_{1} y(t)+\varrho y^{2}(t)+\lambda_{3} y^{3}(t)}_{=\mathscr{F}[y(t)]}=\tilde{u}(t)
$$


where $\mathscr{F}[y(t)]$ is the nonlinear operator responsible for encoding the hysteretic characteristics smoothly, and $\varrho$ is defined as $\varrho=\lambda_{2}\left(\varrho=-\lambda_{2}\right)$ for sign $[\dot{\tilde{u}}(t)]<0$ $(\operatorname{sign}[\dot{\tilde{u}}(t)]>0)$. Additionally, for a single-tone input $\tilde{u}(t)=A e^{j \omega t}$, the term $u_{0}(t)=\lambda_{0} \Phi[\dot{\tilde{u}}(t)]$, which is considered as an additional input applied to the equivalent system, is given by:

$$
u_{0}(t)=\frac{j 2 \lambda_{0}}{\pi} \underbrace{\sum_{k=1}^{2}\left(\frac{e^{-j[1+2(k-1)] \omega t}-e^{j[1+2(k-1)]) \omega t}}{1+2(k-1)}\right)}_{=\Phi[\dot{\tilde{u}}(t)]}
$$

where $\Phi[\dot{\tilde{u}}(t)]$ is the complex exponential Fourier series up to the second term.

Although the methodology proposed for smoothing hysteretic systems was carried out on a Bouc-Wen model, its general form allows investigation of other models. Specifically, on jointed structures, the bounding functions could be formed following the Masing hypothesis, which is an assumption well accepted in the literature to capture friction on interfaces [27, 28.

2.2. Derivation of the higher-order FRFs through the harmonic probing method

For multi-input and single-output (MISO) systems, the functional of the Volterra series in the continuous-time domain is given by [12]:

$$
\begin{aligned}
y(t) & =\sum_{\eta=1}^{\infty} y_{\eta}(t)=y_{0}+\underbrace{\sum_{p=1}^{j} \int_{\mathbb{R}^{1}} h_{1}^{\left(u_{p}\right)}\left(\tau_{1}\right) u_{p}\left(t-\tau_{1}\right) d \tau_{1}}_{=y_{1}(t)} \\
& +\underbrace{\sum_{p=1}^{j} \sum_{k=1}^{j} \int_{\mathbb{R}^{2}} h_{2}^{\left(u_{p}, u_{k}\right)}\left(\tau_{1}, \tau_{2}\right) u_{p}\left(t-\tau_{1}\right) u_{k}\left(t-\tau_{2}\right) d \tau_{1} d \tau_{2}}_{=y_{2}(t)} \\
& +\sum_{p=1}^{\sum_{k=1}^{j} \sum_{l=1}^{j} \int_{\mathbb{R}^{3}} h_{3}^{\left(u_{p}, u_{k}, u_{l}\right)}\left(\tau_{1}, \tau_{2}, \tau_{3}\right) \times} \underbrace{u_{p}\left(t-\tau_{1}\right) u_{k}\left(t-\tau_{2}\right) u_{l}\left(t-\tau_{3}\right) d \tau_{1} d \tau_{2} d \tau_{3}}_{=y_{3}(t)}
\end{aligned}
$$

and higher-order contributions

where each polynomial contribution of $\eta$-order is given by :

$$
\begin{aligned}
y_{\eta}(t) & =\sum_{p=1}^{j} \sum_{k=1}^{j} \ldots \sum_{n=1}^{j} \int_{\mathbb{R}^{\eta}} h_{\eta}^{\left(u_{p}, u_{k}, \ldots, u_{n}\right)}\left(\tau_{1}, \tau_{2}, \ldots, \tau_{\eta}\right) \\
& \times \prod_{i=1}^{\eta} u_{p}\left(t-\tau_{1}\right) u_{k}\left(t-\tau_{2}\right) \ldots u_{n}\left(t-\tau_{\eta}\right) d \tau_{1} d \tau_{2} \ldots d \tau_{\eta}
\end{aligned}
$$


where $y_{\eta}(t)$ is each contribution of the total output $y(t)$, and the term $h_{\eta}^{\left(u_{p}, u_{k}, \ldots, u_{n}\right)}\left(\tau_{1}, \tau_{2}, \ldots, \tau_{\eta}\right)$ is called Volterra kernel related to the $j^{\text {th }}$ input. The multi-dimensional Fourier transform of the Volterra kernels for multi-inputs can be calculated by [29]:

$$
\begin{array}{r}
\mathcal{H}_{\eta}^{\left(u_{p}, u_{k}, \ldots, u_{n}\right)}\left(\omega_{1}, \omega_{2}, \cdots, \omega_{\eta}\right)=\int_{\mathbb{R}^{\eta}} h_{\eta}^{\left(u_{p}, u_{k}, \ldots, u_{n}\right)}\left(\tau_{1}, \tau_{2}, \ldots, \tau_{\eta}\right) \times \\
\prod_{i=1}^{\eta} e^{-j \omega_{i} \tau_{i}} d \tau_{1} d \tau_{2} \ldots \tau_{\eta}
\end{array}
$$

where $\mathcal{H}_{\eta}^{\left(u_{p}, u_{k}, \ldots, u_{n}\right)}\left(\omega_{1}, \omega_{2}, \cdots, \omega_{\eta}\right)$ are the higher-order frequency response functions, which can be used to describe the system output for any input with a well-known mathematical expression. To simplify the notation, it is assumed that:

$$
\int_{\mathbb{R}^{\eta}}=\int_{\mathbb{R} \times \mathbb{R} \times \ldots \mathbb{R}}=\underbrace{\int_{-\infty}^{\infty} \int_{-\infty}^{\infty} \cdots \int_{-\infty}^{\infty}}_{\eta \text { th order }}
$$

Additionally, the computation of the analytical expressions for each higherorder FRFs is carried out through the harmonic probing method. For a threetone harmonic input given by:

$$
\begin{array}{r}
u(t)=A \cos \omega_{1} t+B \cos \omega_{2} t+C \cos \omega_{3} t \\
\Rightarrow u(t)=\frac{A\left(e^{j \omega_{1} t}+e^{-j \omega_{1} t}\right)}{2}+\frac{B\left(e^{j \omega_{2} t}+e^{-j \omega_{2} t}\right)}{2}+\frac{C\left(e^{j \omega_{3} t}+e^{-j \omega_{3} t}\right)}{2}
\end{array}
$$

the generalized expression for the contributions mapping of $\eta$-order is written as [30]:

$$
\begin{aligned}
& y_{\eta}(t)= \\
& \frac{1}{2^{\eta}} \sum_{N+M+L=\eta} A^{N} B^{M} C^{L}\left(C_{p, q, r, s, v, \varpi}^{\eta}\right) \mathcal{H}_{\eta}^{p, q, r, s, v, \varpi}(\omega) e^{j\left(N \omega_{1}+M \omega_{2}+L \omega_{3}\right) t}
\end{aligned}
$$

where $p, q, r, s, v$ and $\varpi$ are integer values and

$$
\begin{array}{r}
C_{p, q, r, s, v, \varpi}^{\eta}=\frac{\eta}{p ! q ! r ! s ! v ! \varpi !} \\
\mathcal{H}_{\eta}^{p, q, r, s, v, \varpi}(\omega)=\mathcal{H}_{\eta}(\underbrace{\omega_{1}, \ldots, \omega_{1}}_{p \text { times }}, \underbrace{-\omega_{1}, \ldots,-\omega_{1}}_{q \text { times }}, \underbrace{\omega_{2}, \ldots, \omega_{2}}_{r \text { times }}, \underbrace{-\omega_{2}, \ldots,-\omega_{2}}_{s \text { times }}, \\
\underbrace{\omega_{3}, \ldots, \omega_{3}}_{v \text { times }}, \underbrace{-\omega_{3}, \ldots,-\omega_{3}}_{\varpi \text { times }})
\end{array}
$$


The generalized expressions for the harmonic probing algorithm allow deriving analytical solutions of the higher-order FRFs for the equivalent system in equation (14). Then, after establishing the theoretical foundation of the Volterra kernels, the next section presents a complete description of the identification procedure strategy proposed by this paper.

\section{On the Parameter Estimation Procedure through the Higher-Order FRFs and the Bayesian Inference}

\subsection{On the parameter estimation procedure}

The outline of the identification procedure is explained as follows:

- Step 1. Data acquisition:

205

In principle, this step involves experimental measurements considering controlled vibration tests. The following input signals were considered: white-noise signal with low excitation amplitude to estimate parameters related to the linear regime of motion. Probing testing signals were considered to generate the experimental higher-order FRFs. Experimental data from sweeping sine tests were acquired for verification and testing of the proposed Bouc-Wen oscillator.

- Step 2. Linear regime of motion:

This step identifies the underlying linear model. It starts with a modal testing procedure for identification of the parameters $\omega_{n}$ and $\zeta$. To accomplish that, the line-fit method [31] is applied on the experimental FRF, which is estimated when the system is subjected to a white noise input with low excitation level.

The framework of the higher-order FRFs requires the knowledge of the firstorder Volterra kernel, which corresponds to the conventional FRF. Having computed the parameters $\omega_{n}$ and $\zeta$, the first-order Volterra kernel is estimated:

$$
\mathcal{H}_{1}(\omega)=\frac{1}{-\omega^{2}+j 2 \omega \zeta \omega_{n}+\omega_{n}^{2}}
$$

- Step 3. Nonlinear regime of motion:

This step covers the calibration of nonlinear parameters based on the higherorder FRFs for hysteretic systems. It is introduced through the following substeps: 3.1. Harmonic filtering, 3.2. Third-order kernel computation, and 3.3. Updating parameters. However, before proceeding, the important characteristics of the framework involving the higher-order FRFs are addressed.

For conditions where the hysteresis loop is almost closed $\left(y_{0} \approx 0\right)$, the coefficients related to the opening and closening of the loop are null, i. e. $\lambda_{0}=\lambda_{2}=0$, whereas the remaining terms result in:

$$
\begin{array}{r}
\lambda_{1}=\alpha \\
\lambda_{3}=\frac{16 \alpha \delta^{2}}{96}-\frac{35 \alpha \delta}{96 Y}
\end{array}
$$


These equations attend the characteristics of the Bouc-Wen model since they are not a function of the parameter $\gamma$, which is responsible for the opening and closening of the hysteresis loop. Thus, under the condition $\left(y_{0} \approx 0\right)$, the equivalent nonlinear system is rewritten as:

$$
\ddot{y}(t)+2 \zeta \omega_{n} \dot{y}(t)+(k+\alpha) y(t)+\lambda_{3} y^{3}(t)=\tilde{u}(t)
$$

Since the nonlinear system in equation (26) has terms up to the cubic order, it is used $\eta=3$ contributions to compute the higher-order FRFs [16, 17, 32]. Thus, applying the harmonic probing method to map the three-dimensional 235 kernel in frequencies $\omega_{1}, \omega_{2}$ and $\omega_{3}$, the derived higher-order FRF yields:

$$
\mathcal{H}_{3}\left(\omega_{1}, \omega_{2}, \omega_{3}\right)=-\mathcal{H}_{1}\left(\omega_{1}\right) \mathcal{H}_{1}\left(\omega_{2}\right) \mathcal{H}_{1}\left(\omega_{3}\right) \mathcal{H}_{1}\left(\omega_{1}+\omega_{2}+\omega_{3}\right) \lambda_{3}
$$

An alternative way to simplify the algebraic complexity of the higher-order FRFs consists of analyzing only their leading diagonal by letting $\omega_{1}=\omega_{2}=$ $\cdots=\omega_{\eta}=\omega$. The leading diagonals encode the main characteristics of the kernels, such as the sub-harmonic resonances and, additionally, the use of this formulation is suitable for calculating response outputs or even estimating nonlinear parameters [16. Thus, the leading diagonal of the third-order Volterra kernel in Eq. 27) is given by:

$$
\begin{array}{r}
\mathcal{H}_{3}(\omega, \omega, \omega)=-\mathcal{H}_{1}(\omega) \mathcal{H}_{1}(\omega) \mathcal{H}_{1}(\omega) \mathcal{H}_{1}(\omega+\omega+\omega) \lambda_{3} \\
\Leftrightarrow \mathcal{H}_{3}(\omega, \omega, \omega) \equiv \mathcal{H}_{3}(\omega)=-\mathcal{H}_{1}^{3}(\omega) \mathcal{H}_{1}(3 \omega) \lambda_{3}
\end{array}
$$

where the dependence of the kernel $\mathcal{H}_{3}(\omega, \omega, \omega)$ on the first-order kernel $\mathcal{H}_{1}(\omega)$ highlights that the previous step is essential to the advance of the identification

(28) indicates the presence of sub-harmonic in the third-order kernel at frequency $3 \omega$. Furthermore, to simplify excessive mathematical notation, once the kernel in Eq. (28) depends only on the frequency component $\omega$, it is considered the equivalent notation $\mathcal{H}_{3}(\omega, \omega, \omega) \equiv \mathcal{H}_{3}(\omega)$.

\section{- Step 3.1. Harmonic filtering:}

Considering a harmonic excitation of frequency $\omega$, the response output of the equivalent nonlinear system from Eq. 27 can be written through the Volterra series representation using $\eta=3$ contributions [14:

$$
y(t)=|Y(\omega)| \cos \left(\omega t+\phi_{1}\right)+|Y(3 \omega)| \cos \left(3 \omega t+\phi_{3}\right)
$$

where $|Y(\omega)|$ and $|Y(3 \omega)|$ are the amplitudes related to the first and third-order harmonic amplitudes, respectively, whereas the respective phases are given by $\phi_{1}=\angle Y(\omega)$ and $\phi_{3}=\angle Y(3 \omega)$. The harmonic amplitudes can be filtered out from the total experimental response using, for instance, the Fourier filtering. 
By truncating the higher-order components at the third-order harmonic amplitude, the kernel $\hat{\mathcal{H}}_{3}^{\exp }(\omega)$ is estimated by [14]:

$$
\hat{\mathcal{H}}_{3}^{\exp }(\omega) \approx \frac{4 Y(3 \omega)}{\tilde{A}^{3}}
$$

For experimental measuring of the kernel $\hat{\mathcal{H}}_{3}^{\text {exp }}(\omega)$ following the assumption that $y_{0} \approx 0$, it is necessary to select properly a frequency range where the harmonic amplitude is distinctly measurable. Based on the methodology proposed by Chatterjee and Vyas [14, which developed an iterative estimation procedure

through the higher-order FRFs, the third-order harmonic component should be measured around a set of frequencies close to the sub-harmonic component $3 \omega=\omega_{n}$, i. e., $\omega=\frac{\omega_{n}}{3}$, where the harmonic $Y(3 \omega)$ is the most prominent.

Jointed structures carry the amplitude-dependent nonlinearity, having a significant evolution of the frictional dissipation in the vicinity of resonance frequencies, where the structure experiences the highest response amplitudes. Since the amplitude response around the sub-harmonic $\omega=\frac{\omega_{n}}{3}$ assumes smaller values in comparison with the amplitude in the vicinity of $\omega_{n}$, the assumption of hysteresis almost closed $y_{0} \approx 0$ seems to be adequate. The results in the section 4.2 exemplify the harmonic amplitudes measured experimentally.

The updating of parameters $\alpha$ and $\delta$ is carried out considering the experimentally measured kernel $\hat{\mathcal{H}}_{3}^{\exp }(\omega)$ :

$$
\hat{\mathcal{H}}_{3}^{\exp }(\omega)=-\mathcal{H}_{1}^{3}(\omega) \mathcal{H}_{1}(3 \omega) \underbrace{\left(\frac{16 \alpha \delta^{2}}{96}-\frac{35 \alpha \delta}{96 Y}\right)}_{=\lambda_{3}}
$$

where the Bayesian inference strategy, which is addressed in section 3.2 , updates the estimative of such parameters.

The advantage of using the Volterra series at this identification step lies in the possibility of visualizing, with physical meaning, the influence of the nonlinear parameters on the system output through the Volterra contributions. Additionally, the propagation of uncertainties through the analytical formulation of the third-order kernel reduces the computational effort for the Bayesian identification.

- Step 4. Model Verification:

This step conducts the updating, verification, and validation of the nonlinear model. The fourth step of the proposed strategy aims firstly to identify the last parameter, $\gamma$, which controls the opening of the hysteresis loop when the jointed structure operates under a nonlinear regime of motion. Since this is the parameter that remains to be identified, it is estimated through the calibration of the integrated numerical output of the proposed Bouc-Wen model in terms 
of the experimental output of the jointed structure. The model verification is performed through a comparison between the response of the identified model with experimental measurements, whereas the validation occurs comparing the experimental hysteresis loop with the estimated one.

\subsection{Bayesian inference strategy}

We employ the Bayesian framework to infer information about the system parameters. In this framework, prior knowledge is updated with experimental data, statistical envelopes can be obtained for the identified parameters and the model response, and it is possible to compute correlation among the variables.

295 The procedure considers that: (1) the parameters to be identified are modeled as random variables $\boldsymbol{\theta} \in \mathbb{R}^{N}$, and the system observations are also random $\mathscr{D} ;(2)$ a prior probability density function is assigned to the random vector (composed of random variables), and a likelihood function is obtained from some hypotheses of the process; (3) the solution lies in updating the prior density function based on a set of data observations $\mathscr{D}$ from the system or structure in interest, which converts an a priori density function into a posteriori one $\pi(\boldsymbol{\theta} \mid \mathscr{D})$ 33, 34 .

In this sense, having $\mathscr{D}$ and $\boldsymbol{\theta}$ as random variables, the posterior density function is given based on the Bayes' theorem:

$$
\pi(\boldsymbol{\theta} \mid \mathscr{D})=\frac{\pi(\mathscr{D} \mid \boldsymbol{\theta}) \pi(\boldsymbol{\theta})}{\pi(\mathscr{D})}
$$

where $\pi(\boldsymbol{\theta} \mid \mathscr{D})$ is the updated posterior probability density function given the system observations $\mathscr{D}, \pi(\mathscr{D} \mid \boldsymbol{\theta})$ is the likelihood function, $\pi(\boldsymbol{\theta})$ is the prior probability density function containing evidences for candidate model parameters, whereas $\pi(\mathscr{D})$ is a normalizing constant that ensures $\pi(\boldsymbol{\theta} \mid \mathscr{D})$ is a probability density function with integral equal to unity.

Assuming that the fluctuations in the system response are related to the measurement process, the measurement data $\mathscr{D}$ are described as:

$$
\mathscr{D}=\mathscr{D}^{\mathcal{M}}(\boldsymbol{\theta})+\varepsilon
$$

where $\mathscr{D}^{\mathcal{M}}(\boldsymbol{\theta})$ corresponds to model $\mathcal{M}$ predictions, given a set of parameters $\boldsymbol{\theta}$, and $\varepsilon \sim \mathcal{N}\left(\mathbf{0}, I \sigma_{\varepsilon}^{2}\right)$ is a random noise vector $\varepsilon \in \mathbb{R}^{N}$ with covariance $I \sigma_{\varepsilon}^{2}$, where $I$ is the identity matrix, i.e., we are assuming and additive decorrelated Gaussian noise. Based on equation (33), the analytical expression of the likelihood function yields:

$$
\pi(\mathscr{D} \mid \boldsymbol{\theta}) \propto \exp \left(-\frac{1}{2} \frac{\left(\mathscr{D}-\mathscr{D}^{\mathcal{M}}(\boldsymbol{\theta})\right)^{T}\left(\mathscr{D}-\mathscr{D}^{\mathcal{M}}(\boldsymbol{\theta})\right)}{\sigma_{\varepsilon}^{2}}\right)
$$

and a common point estimate $\boldsymbol{\theta}^{*}$ is the maximum a posteriori probability (MAP):

$$
\boldsymbol{\theta}^{*}=\arg \max _{\boldsymbol{\theta}} \pi(\boldsymbol{\theta} \mid \mathscr{D})
$$


It is noteworthy that, if a Uniform prior probability density function $\pi(\boldsymbol{\theta})$ is chosen, the posterior probability density function is proportional to the likelihood function.

320 Toward this background, the parameters $\boldsymbol{\theta}=\{\zeta, k, \alpha, \delta, \gamma\}^{T}$ for modelling the jointed structure are set as the random variables with an Uniform prior probability density function, $\mathscr{D}$ consists of experimental measurements acquired from the jointed structure at Step 1., Step 2. provides an initial estimate for $\omega_{n}$ and $\zeta$, whereas $\mathscr{D}^{\mathcal{M}}(\boldsymbol{\theta})$ is given according to Steps 3. and 4. respectively, 325 which results in the following likelihood expressions:

$$
\begin{array}{r}
\pi\left(\mathscr{D} \mid\{\zeta, \alpha, \delta\}^{T}\right) \propto \exp \left(\frac{-\left\|\mathcal{H}_{3}\left(\{\zeta, \alpha, \delta\}^{T} ; \omega\right)-\hat{\mathcal{H}}_{3}^{\exp }(\omega)\right\|^{2}}{2 \sigma^{2}}\right) \\
\pi(\mathscr{D} \mid \gamma) \propto \exp \left(\frac{-\left\|y(\gamma ; t)-\hat{y}^{\exp }(t)\right\|^{2}}{2 \sigma^{2}}\right)
\end{array}
$$

where $\mathcal{H}_{3}\left(\{\zeta, \alpha, \delta\}^{T} ; \omega\right)$ and $y(\gamma ; t)$ are the leading diagonal of third-order kernel and the output response, respectively, both predicted through the computational model, whereas $\hat{\mathcal{H}}_{3}^{\exp }(\omega)$ and $\hat{y}^{\exp }(t)$ are experimental measurements. Once the prior density function is Uniform, and we are assuming an additive Gaussian noise, the MAP (35) produce the same value as the least squares estimate.

The posterior distribution is approximated numerically. Samples are generated from the Markov Chain Monte Carlo (MCMC)/Metropolis-Hastings algorithm [35, 36]. The random variables $\boldsymbol{\theta}$ are limited to the interval $\left[\boldsymbol{\theta}_{\min }, \boldsymbol{\theta}_{\max }\right]$, whose current state is symmetrically normalized as $\boldsymbol{\theta}^{\prime}=(1-\boldsymbol{x}) \boldsymbol{\theta}_{\min }+\boldsymbol{x} \boldsymbol{\theta}_{\max }$, 335 and $\boldsymbol{x}$ is a random variable $\in[0,1]$. Only $90 \%$ of the Monte Carlo simulations $n_{s}$ are considered in the final stationary Markov chain (burn-in of 10\%), and the value of the random walk step $\sigma_{p}$ is chosen such that the acceptance rate is about $50 \%$.

Finally, Figure 2 summarizes the strategy proposed along the section, depicting each step into a flowchart representation. 


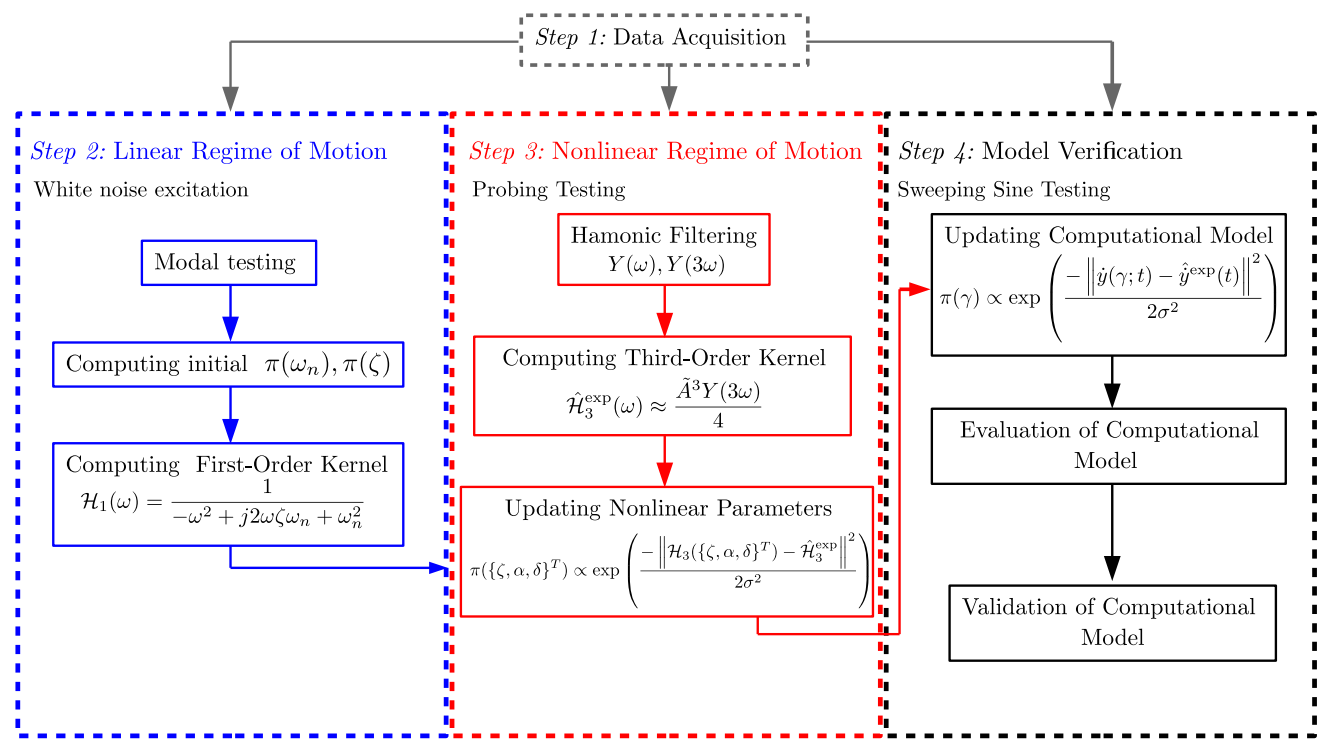

Figure 2: Flowchart of the estimation procedure. Step (1) provides experimental measurements for each next step. Step (2) computes values for $\omega_{n}$ and $\zeta$ and provides prior knowledge about them; in Step (3), after computing the experimental third-order kernel, the PDFs of $\{\zeta, \alpha, \delta\}^{T}$ are updated, including the PDF $\pi(\zeta)$ because of the uncertainty regarding experimental visualizations on the parameter, whereas $\omega_{n}$ is held at the mean value; estimated MAP of $\left\{\zeta, \alpha, \delta, \omega_{n}\right\}^{T}$ are used to construct the computational model in Step (4) and update $\pi(\gamma)$, then to evaluate and to validate the constructed model.

\section{Experimental Application of the Proposed Methodology in a Bolted Joint Structure}

\subsection{Experimental setup}

The identification problem tackled may be stated as follows: the experimental test rig is nonlinearly dependent on excitation amplitude, presenting the well-known softening effect of friction joints. The structure presents data variation related to the presence of uncertainties in the measurement process. The experimental measurements were conducted through different days and only the tightening torque in the joint connection was controlled after each experimental 350 realization. Once the general aspects of the identification problem have been discussed, this subsection focus on describing the experimental setup.

Figure 3 presents the jointed structure investigated. The test rig is formed of two aluminum beams, each one with dimensions of $270 \times 25.4 \times 6.35 \mathrm{~mm}$ and assembled by a bolted-joint connection with a tightening torque of $5 \mathrm{Nm}$.

355 The excitation of the structure was conducted by a Modal Shop 2400E shaker located at $85 \mathrm{~mm}$ from the clamped end of the cantilever beam to minimize shaker-structure interaction [37. Due to observability purposes for modeling the first vibration mode of the structure, the system output was measured at the free end of the beam, using a laser vibrometer Polytec $($ OFV-525/5000S. 
The data acquisition system was the LMS SCADAS. The structure was excited using different levels of input amplitude $0.05 \mathrm{~V}$ (low), $0.10 \mathrm{~V}$ (medium) and $0.20 \mathrm{~V}$ (high). This paper regards the voltage supplied by the shaker amplifier as the excitation signal since it is easier to keep this signal constant over a frequency range. The same strategy was used in Villani et al. 38. All signals were measured with a sampling frequency of $1024 \mathrm{~Hz}$.

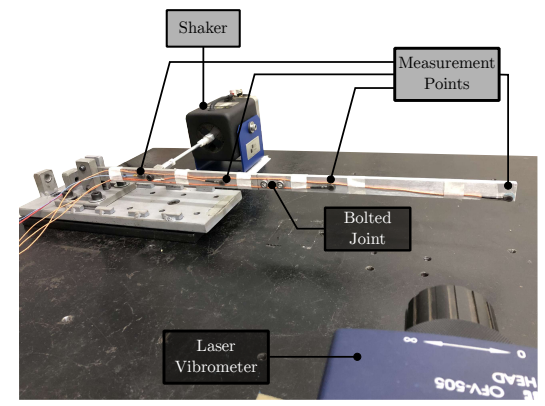

Top view of the bolted joint:

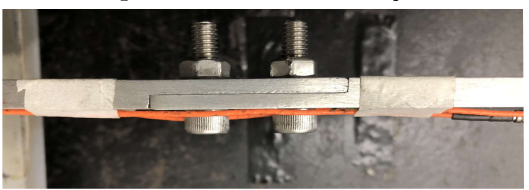

(a) Experimental setup

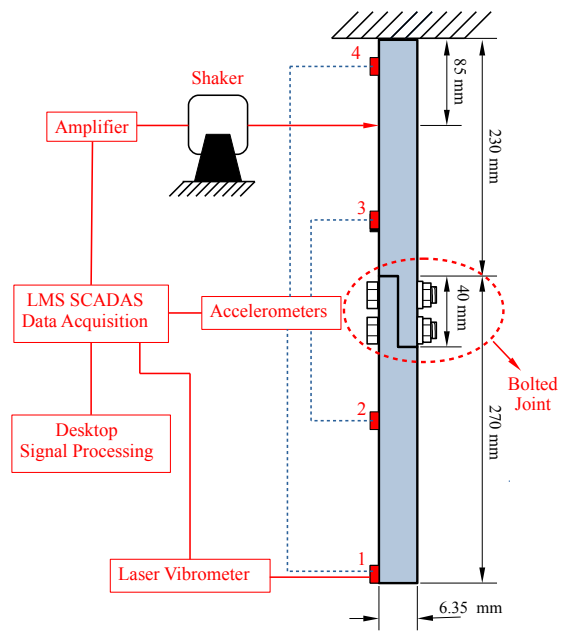

(b) Schematic top view of the experimental setup.

Figure 3: Experimental setup and the schematic representation illustrating the cantilever beam which carries a bolted joint connection.

Figure 4(a) illustrates the receptance calculated during a sweeping sine test from 0 to $40 \mathrm{~Hz}$ (around the first mode), collected with 16384 samples and burst of $50 \%$, regarding different input levels supplied in the shaker amplifier. On the receptance, it is worthy of remark that the FRF starts to exhibit distortions and shifts in the resonant peak as the input force level increases. Further, Figure 4 (b) depicts a zoom on the frequency response curve for a stepped sine test from 3 up to $23 \mathrm{~Hz}$, which indicates that the resonant frequency decreases when the forcing amplitude increases, revealing the bolted joint softening operation. For capturing this nonlinear behavior, the Bouc-Wen model parameters must accomplish the condition $0<\gamma \leqslant \delta$.

Figure 5 exemplifies the data fluctuation considering several experimental measurements with $99 \%$ of statistical confidence bands. This figure demonstrates that even with a small confidence band variation, it is necessary to create a stochastic model to encode the observed variations in experimental realizations. 


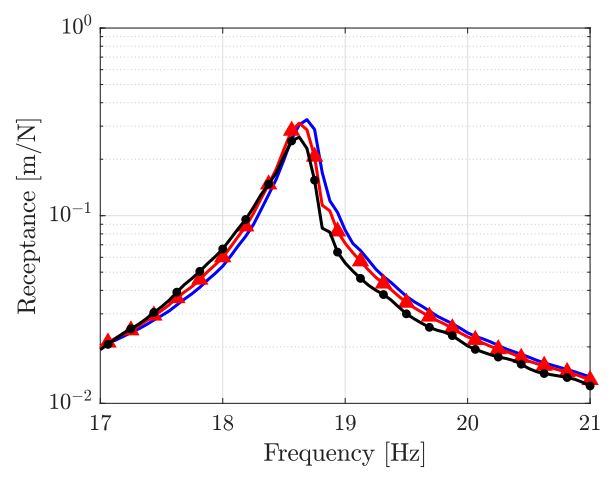

(a) Magnitude plot of the receptance.

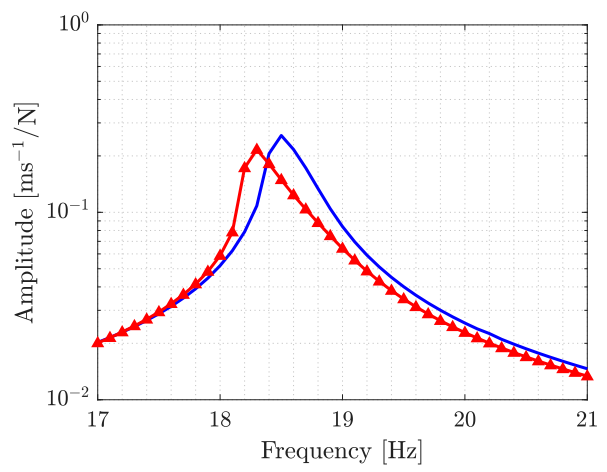

(b) Experimental frequency response curve for a stepped sine test.

Figure 4: Frequency response plots for different excitation amplitudes: —— for low amplitude level $(0.05 \mathrm{~V}), \triangle$ for medium amplitude level $(0.10 \mathrm{~V})$, and $\circ$ for high amplitude level $(0.20$ $\mathrm{V})$.

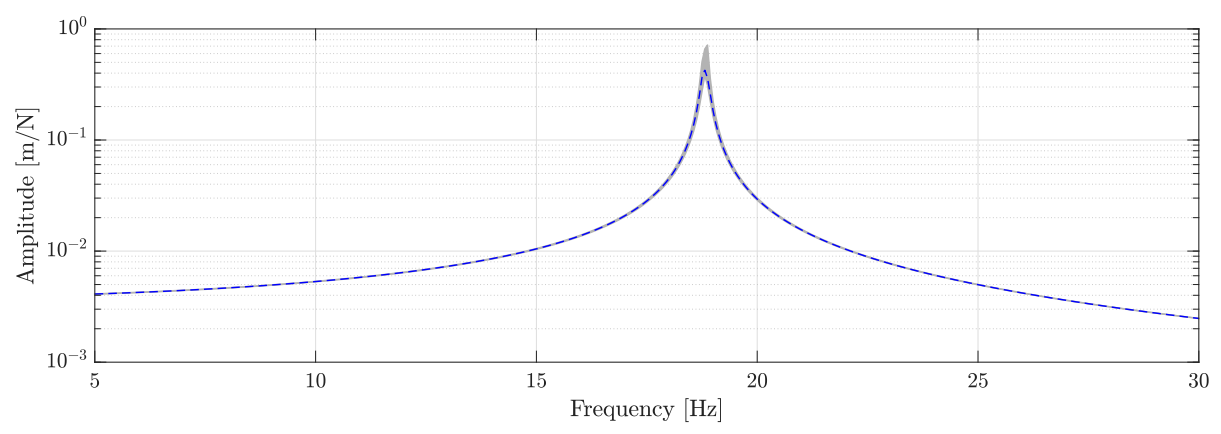

(a) Low level of excitation amplitude $(0.05 \mathrm{~V})$.

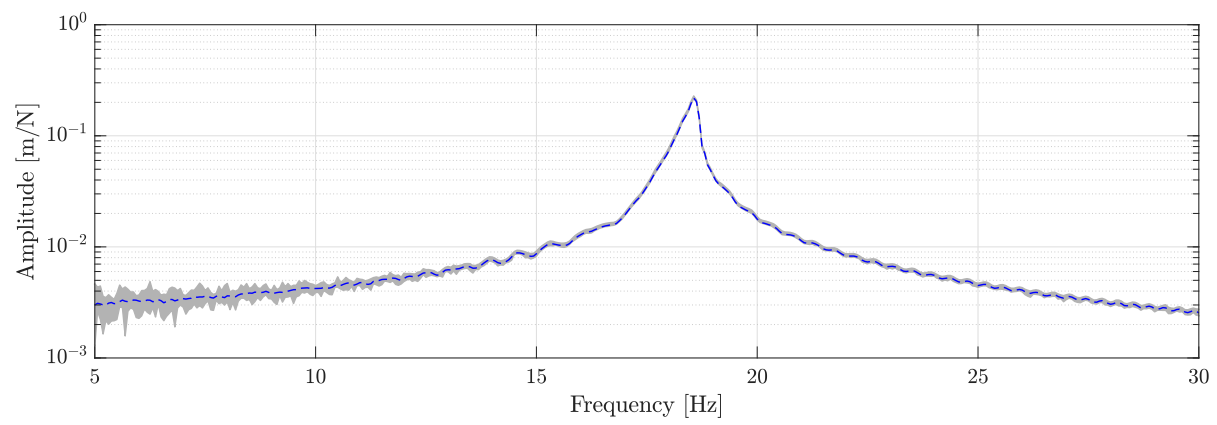

(b) High level of excitation amplitude $(0.20 \mathrm{~V})$.

Figure 5: Variation of the Frequency Response Function calculated for different excitation amplitudes with $99 \%$ of confidence bands. represents the confidence bands, whereas — is the mean values. 


\subsection{Identification of the stochastic Bouc-Wen model}

Based on the identification steps in Fig. 2, this subsection is presented as follows:

- Step 2. Linear regime of motion:

$385 \quad$ Figure 5 (a) represents the FRF obtained experimentally used for extracting the system modal parameters considering a white noise as input signal with low excitation amplitude $(0.05 \mathrm{~V})$. Under this operating condition, the system behaves linearly, and the mean values were $\mu_{\omega_{n}}=18.80 \mathrm{~Hz}$ and $\mu_{\zeta}=0.39 \%$, whereas the coefficient of variation $(\mathrm{CV})$, defined as standard deviation over the mean of the random variables, is $0.176 \%$ and $24.75 \%$, respectively, implying that the uncertainty on the damping ratio is more significant than in the resonance frequency. In this scenario, for the next identification step, the resonance frequency will be held at the mean value of $18.80 \mathrm{~Hz}$, whereas the posterior probability density function of $\zeta$ will be computed through the Metropolis-Hastings algorithm.

- Step 3. Nonlinear regime of motion:

For the identification Step 3.1., Figure 6 exemplifies a set of harmonic amplitudes extracted from the structure response around the sub-harmonic $\omega_{n} / 3$ using Fourier filter. Note that around the selected frequencies of $[5,5.5,6,6.5,6.75]$ $\mathrm{Hz}$, considering a probing input with an amplitude level of $0.15 \mathrm{~V}$ to compute the leading diagonal of the third-order Volterra, the harmonic component $Y(3 \omega)$ is prominent and measurable. The probing signals were collected with 65536 samples to ensure that the harmonic components are extracted during the steady-state condition. As summarized in Step 3.2., the third-order harmonics are considered to compute the experimental third-order kernel.

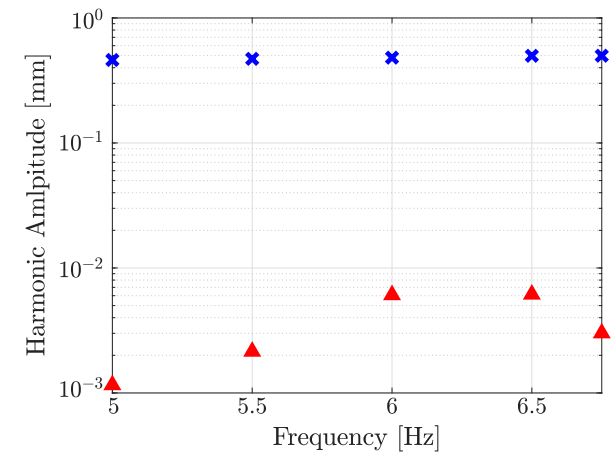

Figure 6: Harmonic Amplitude filtered from the system output. $\triangle$ for $Y(3 \omega)$ and $*$ represents the $Y(\omega)$ amplitude.

For updating the random variables $\boldsymbol{\theta}=\{\zeta, \alpha, \delta\}^{T}$ in Step 3.3., the prior $\mathrm{PDF}$ is set to Uniform assuming as limits $\boldsymbol{\theta}_{\text {min }}=\{0.352 \%, 424.31,272.80\}^{T}$ and $\boldsymbol{\theta}_{\max }=\{0.528 \%, 589.61,409.92\}^{T}$, and then the updated PDF is computed simulating a Markov Chain. In addition, it is important to point out that the 
prior PDF to $\zeta$ is set Uniform such that the previous results identified from Step 2. do not bias the updating at this step. The interval for each parameter was based on initial results considering the sequential quadratic programming (SQP) algorithm [39] as an optimization procedure for minimizing the objective function:

$$
\min J\left(\{\zeta, \alpha, \delta\}^{T}\right)=\left\|\mathcal{H}_{3}\left(\{\zeta, \alpha, \delta\}^{T} ; \omega\right)-\hat{\mathcal{H}}_{3}^{\exp }(\omega)\right\|^{2}
$$

415 subject to:

$$
\begin{array}{r}
0<\alpha \leqslant \omega_{n}^{2} \text { and } \alpha+k=\omega_{n}^{2} \\
0<\delta \leqslant 500
\end{array}
$$

For estimating the Markov Chain, it was assumed $\sigma=6.8092 \times 10^{-9}$, which is calculated based on the covariance matrix of 8 experimental measurements collected at each selected frequency, and $\sigma_{p}=2.5108 \times 10^{-3}$ such that the acceptance rate of the chain was $\sim 50 \%$. The statistics of the parameters were estimated considering Monte Carlo simulations with 850 samplings. The convergence concerning the number of samplings was estimated considering a function which depends on the leading diagonal of the Volterra kernel:

$$
\operatorname{conv}\left(n_{s}\right)=\frac{1}{n_{s}} \sum_{i=1}^{n_{s}} \int_{B}\left\|\mathcal{H}_{3}^{i}(\omega)\right\|^{2} d \omega
$$

where $\mathcal{H}_{3}^{i}$ corresponds to the $i^{\text {th }}$ calculated realization of the Volterra kernel in the Markov Chain, and $B$ is the integration domain.

Figures 7(a), 7(b) and 7(c) show the prior Uniform PDF of $\zeta, \alpha$ and $\delta$ together with the updated (posterior) PDF, respectively. Special attention should be given for Figure 7(c), which shows that $\zeta$ has a completely different PDF than the first one estimated. Besides, it is interesting to note that both PDF of the damping ratio and the $\delta$ parameter exhibit bimodal behavior. The characteristic of these PDFs shows that the Bayesian inference paradigm is robust to the presence of multiple possible solutions for the Bouc-Wen model, in which a deterministic approach is not capable of dealing. Additionally, the prior PDF for each parameter starts with a CV $\sim 28 \%$, whereas the CVs based on the posterior PDFs and given in Table 1 present lower values. Table 1 also shows 435 that the MAP estimator is very close to the mean values for each parameter.

\begin{tabular}{cccc}
\hline Parameter & MAP estimator & $\mu$ & $\mathrm{CV}(\%)$ \\
\hline$\zeta$ & $0.446 \%$ & $0.444 \%$ & 0.57 \\
$\alpha$ & $491.36\left[\mathrm{~N} / \mathrm{mkg}^{-1}\right]$ & $497.40\left[\mathrm{~N} / \mathrm{mkg}^{-1}\right]$ & 0.98 \\
$\delta$ & 341 & 340.21 & 0.98 \\
\hline
\end{tabular}

Table 1: MAP estimator, mean value $\mu$ and $\mathrm{CV}$ for each random variable.

An alternative way to quantify how much information is lost concerning 
the prior PDF is computing the Kullback-Leibler (KL) Divergence [40]. The KL divergence measures the similarity between two PDFs and is useful to analyze which PDF provides a better representation of the experimental observations. For each posterior PDF at this step, the divergence values are around $D_{k l}$ (posterior $\mid$ prior $) \approx 0.36$, which indicates that for all cases, the posterior PDFs have added a considerable amount of information in comparison with the Uniform one.

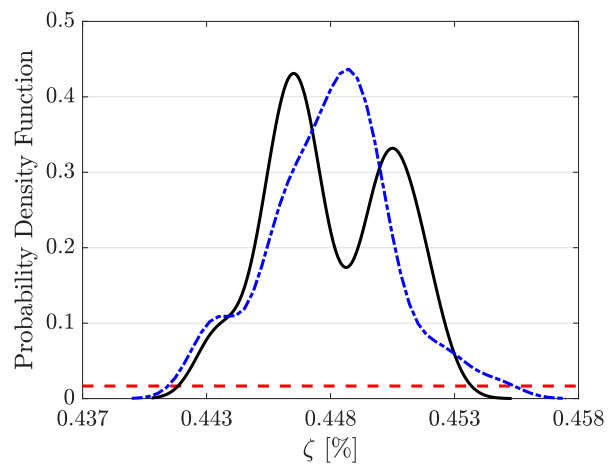

(a) PDF of the random variable $\zeta$.

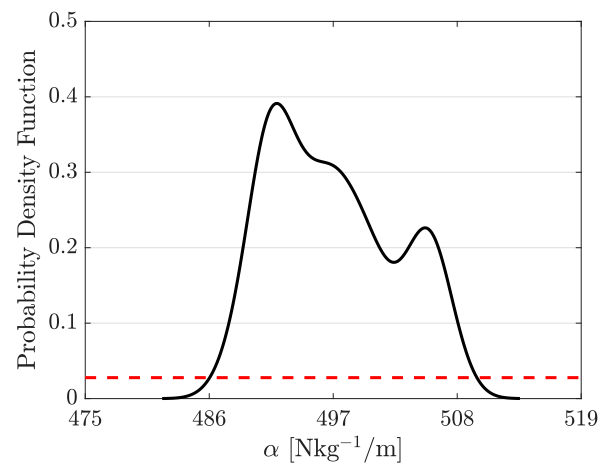

(b) PDF of the random variable $\alpha$.

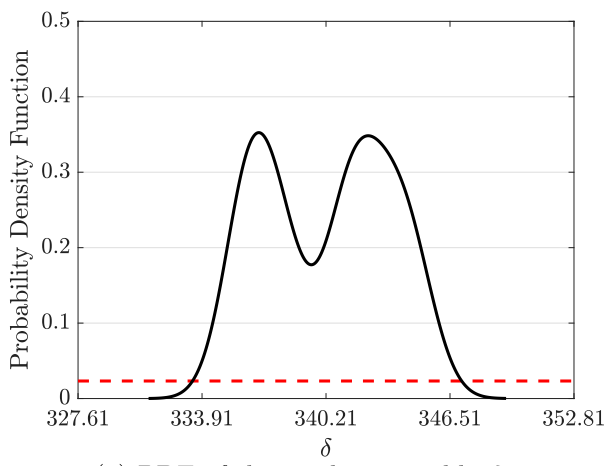

(c) PDF of the random variable $\delta$.

Figure 7: PDF of the random variables $\boldsymbol{\theta}=\{\zeta, \alpha, \delta\}^{T}$. In (a), - - represents the PDF estimated after modal analysis, whereas in (a), (b) and (c), - - - is the Uniform PDF and is the posterior one.

Figure 8 depicts the leading diagonal of the calibrated third-order Volterra capture the data variation in experimental measurements. 


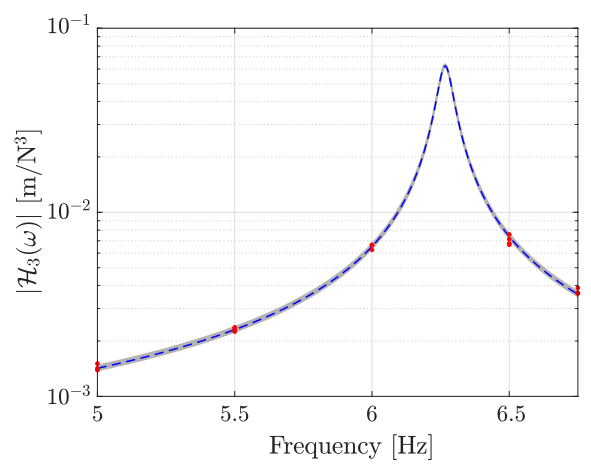

(a) Third-order Volterra kernel.

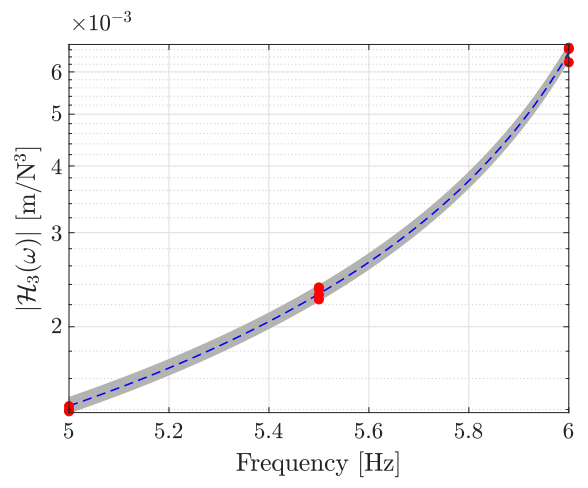

(b) Zoom between 5 and $6 \mathrm{~Hz}$.

Figure 8: Comparison between the leading diagonal of the third-order Volterra kernel estimated versus the experimental data. represents the $99 \%$ model response confidence bands, — is the model response mean and $\bullet$ represents three experimental realizations.

- Step 4. Model verification:

The Step 4. of the identification procedure starts estimating the $\gamma$ parameter. At this stage, once the CVs of the parameters identified earlier are low $\sim 1 \%$, they were held fixed at their mean values $\{\zeta, \alpha, \delta\}^{T}=\{0.444 \%, 497.40,320.21\}^{T}$, just as the resonance frequency. Thus, the prior PDF is also set to Uniform assuming the interval $\gamma_{\min }=17$ and $\gamma_{\max }=41$ based on initial observations performed on the following objective function solved through the SQP algorithm:

$$
\min J(\gamma)=\frac{\left\|\hat{\dot{y}}^{\exp }(t)-\dot{y}(\gamma ; t)\right\|}{\left\|\hat{\dot{y}}^{\exp }(t)-\operatorname{mean}(\hat{\dot{y}} \exp (t))\right\|}
$$

subject to:

$$
0<\gamma \leqslant \delta
$$

where $[y(\gamma ; t) \dot{y}(\gamma ; t) \quad \ddot{y}(\gamma ; t)]^{T}$ are obtained through a numerical integration scheme with the $4^{\text {th }}$ order Runge-Kutta method and variable time-step, whereas the collected testing data (velocity vibration signal measured by the laser vibrometer Figure 30 $\dot{y}^{\exp }(t)$ for this step is based on a sweeping sine test around the first vibrating mode, from 0 to $40 \mathrm{~Hz}$, with an amplitude level of $0.20 \mathrm{~V}$ supplied in the shaker amplifier.

For estimating the posterior PDF of $\gamma$ parameter, the Markov Chain was computed assuming $\sigma=1.26 \times 10^{3}$, which is based on the covariance of 48 experimental measurements, and $\sigma_{p}=0.025$, ensuring an acceptance rate also ${ }_{465}$ around $50 \%$. Besides, the statistics of $\gamma$ was also estimated considering Monte Carlo simulations with 850 samplings. For this step, the convergence was estimated based on equation 40 and the numerically integrated velocity of the 
Bouc-Wen model considering high level of excitation amplitude.

Figure 9 presents a comparison between the prior and posterior PDFs of $\gamma$ parameter. Similar to what happened in the previous step, the posterior PDF also enclosed a great amount of information around the mean, providing a mean value of $\mu_{\gamma}=32.84$, whereas the MAP estimated $\gamma^{*}=33.01$. The CV of the posterior PDF is greater than in previous cases, being $10.20 \%$ but lower than the $\mathrm{CV}$ of the prior PDF, which starts $\sim 28 \%$. Further, the KL divergence for the $\gamma$ parameter is $D_{k l}$ (posterior $\|$ prior $)=1.236$, which means that in this specific case, the information lost by using the Uniform approximation is greater than in the other ones.

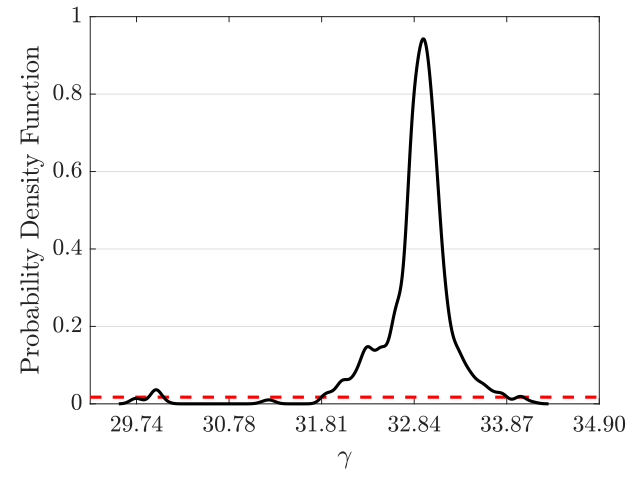

Figure 9: PDF of the random variable $\gamma .---$ represents the prior PDF, whereas — is the posterior one.

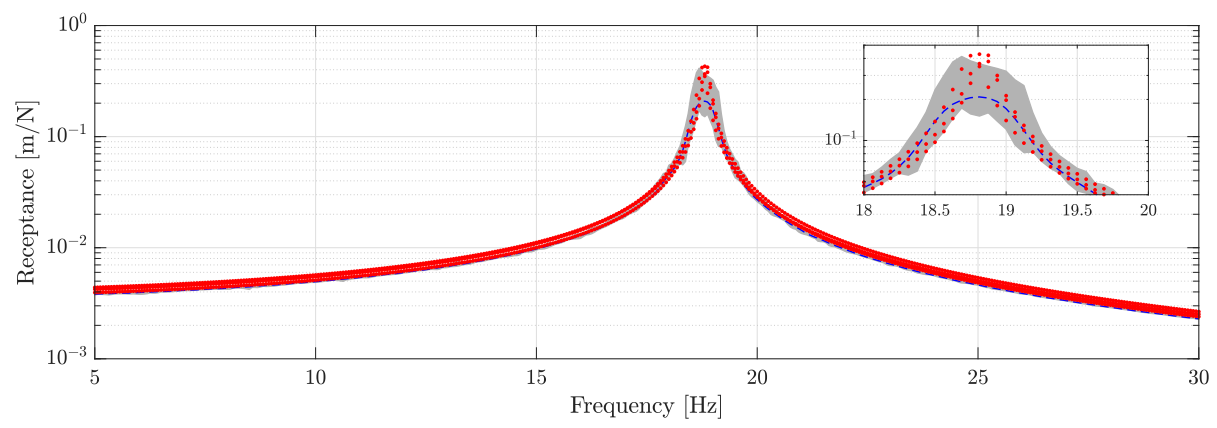

Figure 10: Comparison between the frequency response functions estimated from the numerical Bouc-Wen versus the experimental data around the first vibrating mode. represents the $99 \%$ model response confidence bands, $\_$is the model response mean and $\bullet$ represents three experimental realizations.

For evaluation of the stochastic Bouc-Wen model, Figure 10 presents a comparison between the FRF reproduced by the Bouc-Wen with $99 \%$ of statistical bands and three FRFs obtained experimentally considering white-noise excitation at the low amplitude level. As well as the experimental receptance, the computational one also presents more significant uncertainty related to the damping. 


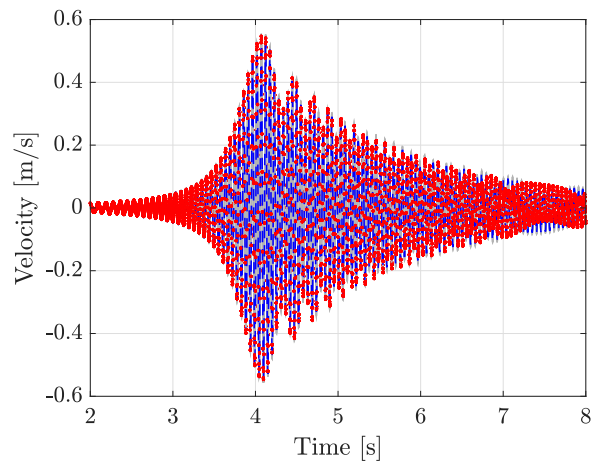

(a) Response output.

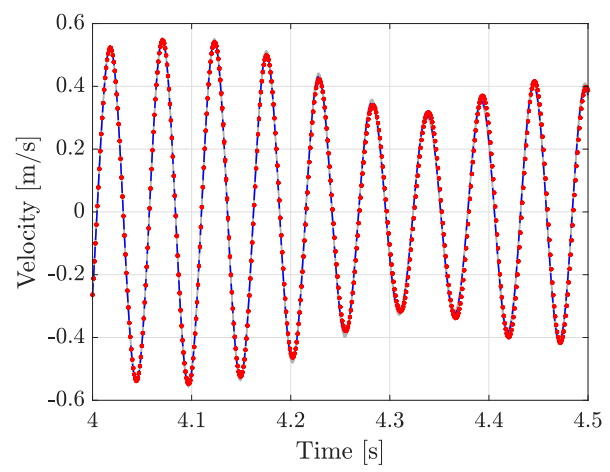

(b) Response zoom between 4 and 4.5 seconds.

Figure 11: Verification and validation of the Bouc-Wen model for low excitation amplitude $(0.05 \mathrm{~V})$ considering a sweeping sine test. represents the $99 \%$ model response confidence bands, $\_$is the model response mean and - represents three experimental realizations.

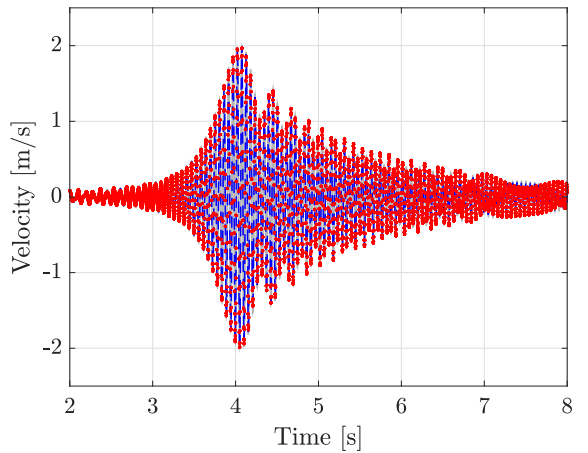

(a) Response output.

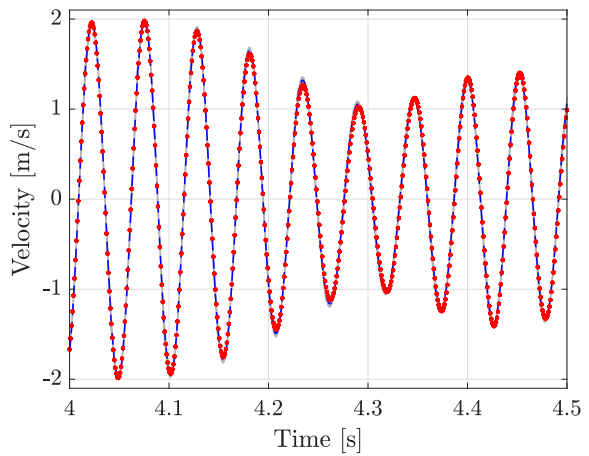

(b) Response zoom between 4 and 4.5 seconds.

Figure 12: Verification and validation of the Bouc-Wen model for high excitation amplitude $(0.20 \mathrm{~V})$ considering a sweeping sine test.

represents the $99 \%$ model response confidence bands, $\_$is the model response mean and • represents three experimental realizations.

Figures 11 and 12 illustrate the predicted response of the numerical model with $99 \%$ of statistical confidence bands in comparison with experimental data for a sweeping sine test with low and high excitation amplitude, respectively. The narrow confidence bands are a feature of the applied computational model, which represents well the experiment under analysis.

Finally, Figure 13 exhibits the measured and the predicted hysteresis loops for different amplitudes applied in the shaker. In all cases, both hysteretic cycles enclose substantially the same area on the restoring force versus displacement plane, producing an adequate prediction for the experimental tests. This figure evidences that the Bouc-Wen model can accommodate enough accuracy of the experimental test-bench. In addition, Figure 13(a) shows that for a maximum displacement of $\sim 4.8 \mathrm{~mm}$, the hysteresis loop is almost closed, which exemplifies that the assumption made during the Step (3) is reasonable, since the maximum 
displacement amplitude in Figure 6 is about $\sim 0.8 \mathrm{~mm}$.

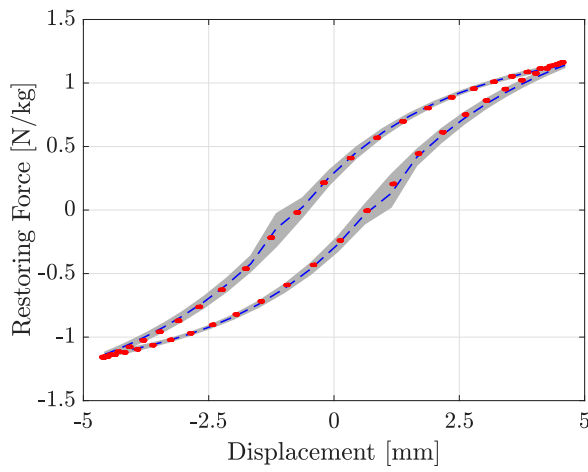

(a) $0.05 \mathrm{~V}$.

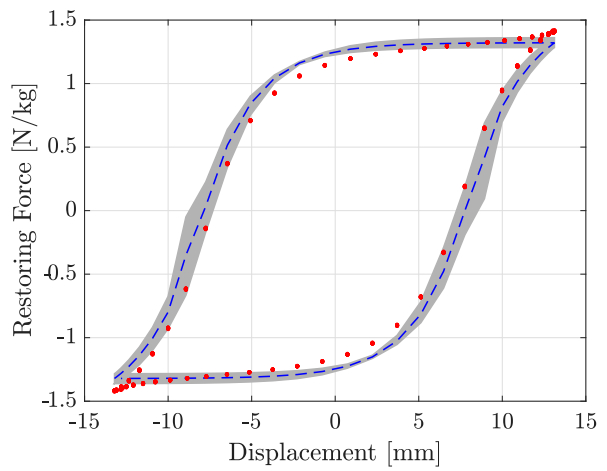

(c) $0.15 \mathrm{~V}$.

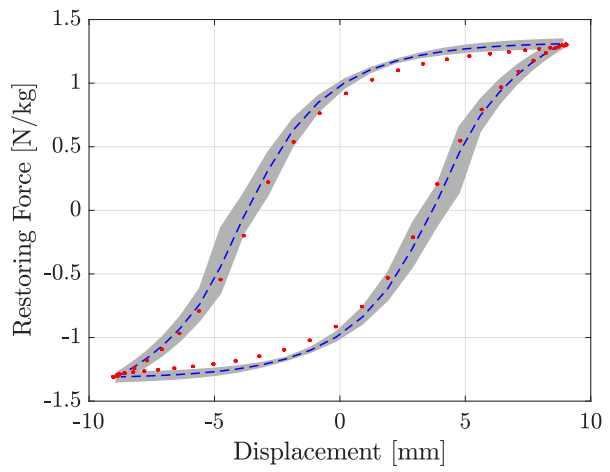

(b) $0.10 \mathrm{~V}$.

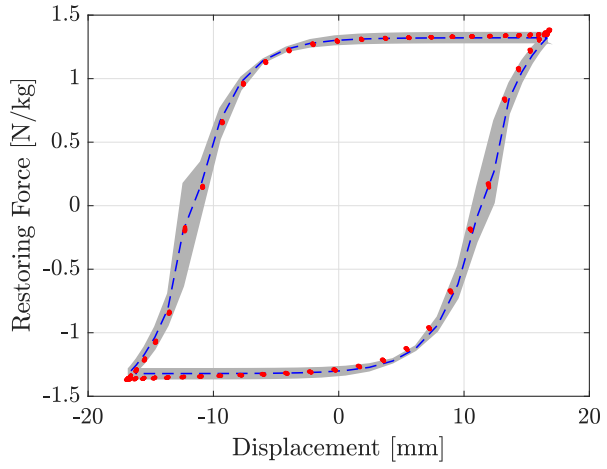

(d) $0.20 \mathrm{~V}$

Figure 13: Comparison between the identified hysteresis loop through the Bouc-Wen versus experimental data for several excitation amplitudes. represents the $99 \%$ model response confidence bands, $\_$is the model response mean and $\bullet$ represents three experimental realizations.

\section{Final Remarks}

This work proposes a procedure to identify structures that have jointed connections based on a four-step algorithm. The identification procedure was applied to an experimental setup under a nonlinear regime of motion due to the frictional contact between different structural components. This work considers the white-box modeling of a Bouc-Wen model for capturing the dynamical behavior of the jointed structure. A set of preliminary experimental tests have shown fluctuations in the system response. Therefore, a Bayesian inference strategy for updating the model parameters was employed. The Bayesian strategy presented in this paper could be used directly to identify the complete Bouc-Wen model, just setting all parameters to update in Step (4), which carries similar particularities to the Worden and Hensman [4] work. However, the 

estimation, providing a clear physical meaning about the structure behavior and conducted with traditional experimental tests.

The results of the stochastic Bouc-Wen model indicates that the computational model can provide an adequate description of assembled structures by fluctuations related to the damping ratio, which were also visualized in the experimental FRF. The computational hysteretic loops carry a full confidence band around the displacement $y_{0}$, especially in Figure 13 (a), where the hysteresis loop is almost closed. Despite this, it was possible to predict with an ${ }_{520}$ adequate agreement the output of the jointed structure considering several excitation amplitudes.

Finally, this work is a natural extension of the methodology developed by Teloli and da Silva [17, exploring the advantages of using the Volterra series as an alternative tool to perform the nonlinear parametric estimation. Although the benchmark addressed here is a controlled experimental laboratory setup and still far from complex industrial cases, this paper presents the use of both higher-order FRFs and the Bouc-Wen model as effective alternatives to deal with problems involving experimental bolted joints with data fluctuation, which is an original contribution to literature. In future work, additional experiments will be 530 collected to test the identification procedure in an even more uncertain scenario, involving different tightening torque conditions and assembly/disassembly of jointed structures, as this is a challenging issue that includes several variabilities on the structures response. Additionally, future works will also focus on using the Bouc-Wen parameters for modeling the contact interfaces in whole-joint 53 element models of assembled structures. To acomplish this task, modifications on the hysteretic model are expected to acommodate the non-local memory effects present in frictional forces according to the pre-sliding and sliding regimes of motion.

\section{Acknowledgments}

The authors are thankful for the financial support provided by São Paulo Research Foundation (FAPESP) Grant number 2016/21973-5, 2017/15512-8 and 2019/06540-3 and CNPq grant number 306526/2019-0.

\section{References}

1. H. Ahmadian, H. Jalali, Identification of bolted lap joints parameters in assembled structures, Mechanical Systems and Signal Processing 21 (2) (2007) 1041 - 1050. doi :https://doi.org/10.1016/j.ymssp.2005.08.015

a URL http://www.sciencedirect.com/science/article/pii/ S0888327005001263

2. Y. Song, C. Hartwigsen, D. McFarland, A. Vakakis, L. Bergman, Simulation 550 of dynamics of beam structures with bolted joints using adjusted Iwan 
beam elements, Journal of Sound and Vibration 273 (1) (2004) 249 - 276.

doi:https://doi.org/10.1016/S0022-460X (03)00499-1.

口

URL http://www.sciencedirect.com/science/article/pii/ S0022460X03004991

3. D. J. Segalman, Modelling joint friction in structural dynamics, Structural Control and Health Monitoring 13 (1) (2006) 430-453. arXiv:https:

//onlinelibrary.wiley.com/doi/pdf/10.1002/stc.119, doi:10.1002/ stc. 119 .

URL https://onlinelibrary.wiley.com/doi/abs/10.1002/stc.119

560 4. H. Ahmadian, H. Jalali, Generic element formulation for modelling bolted lap joints, Mechanical Systems and Signal Processing 21 (5) (2007) 23182334. doi:10.1016/j.ymssp.2006.10.006.

URL https://doi.org/10.1016\%2Fj.ymssp.2006.10.006

5. M. R. Brake, The Mechanics of Jointed Structures: Recent Research and Open Challenges for Developing Predictive Models for Structural Dynamics, Springer, 2017.

6. N. Peyret, J.-L. Dion, G. Chevallier, A framework for backbone 1. experimental tracking : Piezoelectric actuators, stop-sine signal and Kalman filtering, Mechanical Systems and Signal Process-

$570 \quad$ ing 78 (2016) $28-42$, special Issue on Piezoelectric Technologies. doi:https://doi.org/10.1016/j.ymssp.2015.09.020.

1 URL http://www.sciencedirect.com/science/article/pii/ S088832701500415X

7. M. Scheel, S. Peter, R. I. Leine, M. Krack, A phase resonance 575 a approach for modal testing of structures with nonlinear dissipation, Journal of Sound and Vibration 435 (2018) 56 - 73. doi:https://doi.org/10.1016/j.jsv.2018.07.010.

10 URL http://www.sciencedirect.com/science/article/pii/ S0022460X18304486

${ }_{580}$ 8. V. Jaumouillé, J.-J. Sinou, B. Petitjean, An adaptive harmonic bal-

ance method for predicting the nonlinear dynamic responses of mechanical systems - Application to bolted structures, Journal of

1. Sound and Vibration 329 (19) (2010) 4048 - 4067. doi:https: //doi.org/10.1016/j.jsv.2010.04.008. URL http://www.sciencedirect.com/science/article/pii/ S0022460X10002464

9. M. Claeys, J.-J. Sinou, J.-P. Lambelin, R. Todeschini, Experiments and

n numerical simulations of nonlinear vibration responses of an assembly with friction joints - Application on a test structure named "Harmony", Mechanical Systems and Signal Processing 70-71 (2016) 1097 - 1116. doi:https://doi.org/10.1016/j.ymssp.2015.08.024. 
URL

http://www.sciencedirect.com/science/article/pii/ S0888327015003866

10. R. Lacayo, L. Pesaresi, J. Groß, D. Fochler, J. Armand, L. Salles, C. Schwingshackl, M. Allen, M. Brake, Nonlinear modeling of struc-

- tures with bolted joints: A comparison of two approaches based on a time-domain and frequency-domain solver, Mechanical Sys-

tems and Signal Processing 114 (2019) 413 - 438. doi:https:

//doi.org/10.1016/j.ymssp.2018.05.033.

URL http://www.sciencedirect.com/science/article/pii/ S0888327018302875

11. E. Bedrosian, S. O. Rice, The output properties of Volterra systems (nonlinear systems with memory) driven by harmonic and Gaussian inputs, Pro605 ceedings of the IEEE 59 (12) (1971) 1688-1707. doi:10.1109/PROC.1971.

12. M. Schetzen, The Volterra and Wiener Theories of Nonlinear Systems, John Wiley and Sons, 1980.

13. W. J. Rugh, Nonlinear System Theory - The Volterra/Wiener Approach, The Johns Hopkins University Press, 1991.

14. A. Chatterjee, N. S. Vyas, Non-linear parameter estimation with

Volterra series using the method of recursive iteration through harmonic probing, Journal of Sound and Vibration 268 (4) (2003) 657 - 678. doi:https://doi.org/10.1016/S0022-460X (02)01537-7. URL http://www.sciencedirect.com/science/article/pii/ S0022460X02015377

15. A. Chatterjee, Identification and parameter estimation of a bilinear oscillator using Volterra series with harmonic probing, International Journal of Non-Linear Mechanics 45 (1) (2010) 12 - 20. doi:http://dx.doi.org/10.1016/j.ijnonlinmec.2009.08.007.

620 URL http://www.sciencedirect.com/science/article/pii/ S0020746209001632

16. R. Lin, T. Ng, Higher-order FRFs and their applications to the iden-

1 tifications of continuous structural systems with discrete localized nonlinearities, Mechanical Systems and Signal Processing 108 (2018) 326 346. doi:https://doi.org/10.1016/j.ymssp.2018.02.033

n URL http://www.sciencedirect.com/science/article/pii/ S0888327018300980

17. R. de O. Teloli, S. da Silva, A new way for harmonic probing of hysteretic systems through nonlinear smooth operators, Mechanical Systems and Signal Processing 121 (2019) 856 - 875. doi:https://doi.org/10.1016/j.ymssp.2018.11.044. 
URL

http://www.sciencedirect.com/science/article/pii/ S0888327018307659

18. F. Ikhouane, J. Rodellar, Systems with hysteresis: analysis, identification and control using the Bouc-Wen model, John Wiley \& Sons, 2007.

19. M. R. W. Brake, The Next Generation of Joints Research, Springer

1. International Publishing, Cham, 2018, pp. 675-689. doi:10.1007/ 978-3-319-56818-8_37. URL https ://doi .org/10.1007/978-3-319-56818-8_37

${ }_{640}$ 20. M. Oldfield, H. Ouyang, J. E. Mottershead, Simplified models of bolted joints under harmonic loading, Computers \& Structures 84 (1) (2005) 25 33. doi:https://doi.org/10.1016/j.compstruc.2005.09.007.

10 URL http://www.sciencedirect.com/science/article/pii/ S0045794905003184

645 21. M. Ismail, F. Ikhouane, J. Rodellar, The hysteresis Bouc-Wen model, a survey, Archives of Computational Methods in Engineering 16 (2) (2009) 161-188. doi:10.1007/s11831-009-9031-8. URL https ://doi.org/10.1007/s11831-009-9031-8

22. F. F. Real, A. Batou, T. G. Ritto, C. Desceliers, Stochastic modeling for hys650 teretic bit-rock interaction of a drill string under torsional vibrations, Jour口 nal of Vibration and Control 25 (10) (2019) 1663-1672. arXiv:https:// doi.org/10.1177/1077546319828245, doi:10.1177/1077546319828245. URL https://doi.org/10.1177/1077546319828245

23. H. Festjens, G. Chevallier, J. luc Dion, A numerical tool for 655 the design of assembled structures under dynamic loads, International Journal of Mechanical Sciences $75 \quad(2013) \quad 170-177$. doi:https://doi.org/10.1016/j.ijmecsci.2013.06.013.

I URL http://www.sciencedirect.com/science/article/pii/ S0020740313001860

660 24. H. Festjens, G. Chevallier, J. Dion, Nonlinear model order reduction of jointed structures for dynamic analysis, Journal of

1. Sound and Vibration 333 (7) (2014) 2100 - 2113. doi:https: //doi.org/10.1016/j.jsv.2013.11.039.

a URL http://www.sciencedirect.com/science/article/pii/ S0022460X13009930

25. L. P. Miguel, R. de Oliveira Teloli, S. [da Silva], Some practical re17 gards on the application of the harmonic balance method for hysteresis models, Mechanical Systems and Signal Processing 143 (2020) 106842. doi:https://doi.org/10.1016/j.ymssp.2020.106842.

670 URL http://www.sciencedirect.com/science/article/pii/ S0888327020302284 
26. H. Jeffreys, B. Jeffreys, Methods of mathematical physics, Cambridge university press, 1999.

27. M. R. W. Brake, Tutorial: Bolted Joints and Tribomechadynamics, in: G. Kerschen (Ed.), Nonlinear Dynamics, Volume 1, Springer International Publishing, Cham, 2019, pp. 427-431.

28. X.-R. Huang, L. Jézéquel, S. Besset, L. Li, O. Sauvage, Nonlinear modal

n. synthesis for analyzing structures with a frictional interface using a generalized Masing model, Journal of Sound and Vibration 434 (2018) 166 - 191. doi:https://doi.org/10.1016/j.jsv.2018.07.027.

nURL http://www.sciencedirect.com/science/article/pii/ S0022460X18304711

29. K. Worden, G. Manson, G. Tomlinson, A hamonic probing algorithm for the multi-input Volterra series, Journal of Sound and Vibration 201 (1) (1997) 67 -84. doi:http://dx.doi.org/10.1006/jsvi.1996.0746.

a URL http://www.sciencedirect.com/science/article/pii/ S0022460X96907464

30. A. Chatterjee, N. S. Vyas, Non-linear parameter estimation in multidegree-of-freedom systems using multi-input Volterra series, Mechanical Systems and Signal Processing 18 (3) (2004) 457 - 489. doi:https://doi.org/10.1016/S0888-3270(03)00016-5.

a URL http://www.sciencedirect.com/science/article/pii/ S0888327003000165

31. N. M. M. Maia, J. M. M. e Silva, Theoretical and experimental modal analysis, Research Studies Press, 1997.

32. L. G. Villani, S. da Silva, A. Cunha, M. D. Todd, On the detection

1. of a nonlinear damage in an uncertain nonlinear beam using stochastic Volterra series, Structural Health Monitoring 0 (0) (0) 1475921719876086.

a arXiv:https://doi.org/10.1177/1475921719876086, doi:10.1177/ 1475921719876086 . URL https://doi .org/10.1177/1475921719876086

33. P. L. Green, K. Worden, Bayesian and Markov chain Monte Carlo methods for identifying nonlinear systems in the presence of uncertainty, Philosophical Transactions of the Royal Society A: Mathematical, Physical

705 and Engineering Sciences 373 (2051) (2015) 20140405. arXiv:https: //royalsocietypublishing.org/doi/pdf/10.1098/rsta.2014.0405, doi:10.1098/rsta.2014.0405.

a URL https://royalsocietypublishing.org/doi/abs/10.1098/rsta. 2014.0405

710 34. D. Castello, T. Ritto, Quantificação de incertezas e estimação de parâmetros em dinâmica estrutural: uma introdução a partir de exemplos computacionais (2016). 
35. D. Gamerman, H. F. Lopes, Markov chain Monte Carlo: stochastic simulation for Bayesian inference, Chapman and Hall/CRC, 2006.

36. T. G. Ritto, Bayesian approach to identify the bit-rock interaction parameters of a drill-string dynamical model, Journal of the Brazilian Society of Mechanical Sciences and Engineering 37 (4) (2015) 1173-1182. doi:10.1007/s40430-014-0234-z. URL https://doi.org/10.1007/s40430-014-0234-z

37. M. Peeters, G. Kerschen, J. Golinval, Modal testing of nonlinear vibrating structures based on nonlinear normal modes: Experimental demonstration, Mechanical Systems and Signal Processing 25 (4) (2011) 1227 - 1247. doi:https://doi.org/10.1016/j.ymssp.2010.11.006.

1 URL http://wWw.sciencedirect.com/science/article/pii/ S0888327010003821

38. L. G. Villani, S. da Silva, A. Cunha, M. D. Todd, Damage detection

- in an uncertain nonlinear beam based on stochastic Volterra series: An experimental application, Mechanical Systems and Signal Processing 128 (2019) 463 - 478. doi:https://doi.org/10.1016/j.ymssp.2019.03.045.

730 URL http://www.sciencedirect.com/science/article/pii/ S0888327019302250

39. P. E. Gill, E. Wong, Sequential Quadratic Programming Methods, in: J. Lee, S. Leyffer (Eds.), Mixed Integer Nonlinear Programming, Springer New York, New York, NY, 2012, pp. 147-224.

735 40. J. R. Hershey, P. A. Olsen, Approximating the Kullback Leibler Divergence between Gaussian Mixture Models, in: 2007 IEEE International Conference on Acoustics, Speech and Signal Processing - ICASSP '07, Vol. 4, 2007, pp. IV-317-IV-320. doi:10.1109/ICASSP. 2007.366913.

41. K. Worden, J. Hensman, Parameter estimation and model selection for a 740 class of hysteretic systems using Bayesian inference, Mechanical Systems and Signal Processing 32 (2012) 153 - 169, uncertainties in Structural Dynamics. doi:https://doi.org/10.1016/j.ymssp.2012.03.019.

1 URL http://www.sciencedirect.com/science/article/pii/ S0888327012001240 